\title{
Linear-Time Computation of a Linear Problem Kernel for Dominating Set on Planar Graphs
}

\author{
René van Bevern ${ }^{1}$, Sepp Hartung ${ }^{1}$, Frank Kammer ${ }^{2}$, \\ Rolf Niedermeier ${ }^{1}$, and Mathias Weller $^{1}$ \\ 1 TU Berlin \\ 2 Universität Augsburg
}

\begin{abstract}
Over the last years, reduction to a problem kernel, or kernelization for short, has developed into a very active research area within parameterized complexity analysis and the algorithmics of NP-hard problems in general [2]. In a nutshell, a kernelization algorithm transforms in polynomial time an instance of a (typically NP-hard) problem to an equivalent instance whose size is bounded by a function of a parameter. Nowadays, it has become a standard challenge to minimize the size of problem kernels. Consider the following examples:

1. For Feedback Vertex Set (given an undirected graph $G$ and a positive integer $k$, find at most $k$ vertices whose deletion destroys all cycles in $G$ ), there first has been an $O\left(k^{11}\right)$-vertex problem kernel [3], improved to an $O\left(k^{3}\right)$-vertex problem kernel and finally to an $O\left(k^{2}\right)$-vertex problem kernel [5]

2. For Dominating Set on planar graphs (given an undirected planar graph $G$ and a positive integer $k$, find at most $k$ vertices such that each other vertex in $G$ has at least one neighbor in the set of selected vertices), there first was a $335 k$-vertex problem kernel [1], which was further refined into a $67 k$-vertex problem kernel [4], both computable in $O\left(n^{3}\right)$ time.

From the viewpoint of practical relevance, however, also the running times of kernelization algorithms have to be optimized. We show an $O(k)$-size problem kernel for Dominating Set in planar graphs that is computable in $O(n)$ time.
\end{abstract}

\section{Bibliography}

[1] J. Alber, M. R. Fellows, and R. Niedermeier. Polynomial-time data reduction for dominating set. J. ACM, 51(3):363-384, 2004.

[2] H. L. Bodlaender. Kernelization: New upper and lower bound techniques. In Proc. 4th IWPEC, volume 5917 of $L N C S$, pages 17-37. Springer, 2009.

[3] K. Burrage, V. Estivill-Castro, M. R. Fellows, M. A. Langston, S. Mac, and F. A. Rosamond. The undirected feedback vertex set problem has a poly $(k)$ kernel. In Proc. 2nd IWPEC, volume 4169 of LNCS, pages 192-202. Springer, 2006.

[4] J. Chen, H. Fernau, I. A. Kanj, and G. Xia. Parametric duality and kernelization: Lower bounds and upper bounds on kernel size. SIAM J. Comput., 37(4):1077-1106, 2007.

[5] S. Thomassé. A $4 k^{2}$ kernel for feedback vertex set. ACM Trans. Algorithms, 6(3): $32: 1-32: 8,2010$. 